Ann. Zootech., I966, 15 (3), 23I-25I.

\title{
UTILISATION DES POMMES DE TERRE DESHYDRATEES ET FRAICHES DANS L'ALIMENTATION DU PORC EN CROISSANCE, EN COMPARAISON AVEC L'ORGE
}

\author{
Y. HENRY et A. RÉRAT \\ avec la collaboration technique de J.-P. Turc, H. Bousquet, P. Jorro et P. Vaugelade \\ Station de Recherches sur l'Élevage des Porcs, \\ Centre national de Recherches zootechniques, 78-Jouy-en-Josas
}

\section{SOMMAIRE}

L'emploi des pommes de terre déshydratées et fraîches dans l'alimentation du porc en croissance, par référence à un régime à base d'orge, a fait l'objet de deux expériences réalisées, l'une en lots et l'autre en cages de digestibilité. Lorsque les animaux sont nourris suivant un système LEIIMANN modifié comportant l'administration de $1500 \mathrm{~g}$ d'un aliment concentré contenant i 7 p. Ioo de matières azotées totales en complément de pommes de terre ou d'orge à volonté, les meilleures performances de croissance sont réalisées par le lot recevant le régime à base d'orge seule. Cependant, dans le cas d'administration de pommes de terre fraîchement cuites, l'indice de consommation exprimé en $\mathrm{kg}$ de matière sèche par $\mathrm{kg}$ de gain est comparable à celui obtenu avec l'orge et les carcasses sont plus maigres. La consommation de pommes de terre selon ce mode de rationnement représente environ $40 \mathrm{p}$. Ioo de la consommation totale de matière sèche. Par ailleurs, l'introduction de pommes de terre déshydratées dans le régime provoque, par rapport aux tubercules frais, des performances moindres en ce qui concerne à la fois la vitesse de croissance, l'indice de consommation et la qualité des carcasses. La valeur de remplacement du produit déshydraté est de $0,8 \mathrm{~kg}$ d'orge (ou UF) par kg contre $0,27 \mathrm{~kg}$ d'orge par $\mathrm{kg} \mathrm{d}$ a tubercules frais (soit respectivement 0,9 et I, I UF par $\mathrm{kg}$ de matière sèche); on peut toutefois penser que la moindre valeur du produit déshydraté par rapport au produit frais est due au moins partiellement à une préparation défectueuse (absence de lavage des tubercules, surchauffe au moment de la déshydratation).

\section{INTRODUCTION}

L'existence d'excédents plus ou moins importants de pommes de terre dans les zones spécialisées de production a conduit à rechercher une solution commode en vue de leur utilisation en alimentation animale, et plus particulièrement dans 1'alimentation du Porc. C'est ainsi qu'il a été envisagé de procéder à leur déshydratation. Cependant, on peut se demander si une telle technique permet de conserver l'inté- 
gralité de la valeur nutritive du produit initial. La présente étude a précisément pour objet de déterminer la valeur alimentaire des pommes de terre déshydratées et leur limite d'utilisation, en comparaison avec les pommes de terre fraîchement cuites et un aliment concentré complet à base d'orge.

\section{TECHNIQUES EXPÉRIMENTALES ET MATÉRIEL UTILISÉ}

Au cours du premier semestre de l'année 1965 , il a été procédé à deux expériences, la première en lots, la deuxième en cages de digestibilité, portant sur l'étude comparée de l'emploi des pommes de terre déshydratées et fraîches dans l'alimentation du porc en croissance.

Les deux lots de pommes de terre déshydratées et fraîches proviennent d'une même récolte ; il s'agit de grenailles de la variété Bintje. La déshydratation a été réalisée par les soins de l'Institut technique de la Pomme de Terre, à l'aide d'un séchoir à luzerne de type rotatif ; les tubercules, non lavés et broyés à l'état de fines particules de 3 à $5 \mathrm{~mm}$, sont soumis au cours de leur progression dans l'appareil à une température variant entre $600^{\circ} \mathrm{C}$ à l'entrée et $120-15^{\circ} \mathrm{C}$ à la sortie. Le produit obtenu ( $\mathrm{kg}$ pour $5 \mathrm{~kg}$ de tubercules frais) se présente sous la forme d'une poudre grossière de couleur brun noirâtre, qui semble avoir subi un début de calcination à la suite d'une chauffe irrégulière. Après passage dans un broyeur à marteaux, ce produit est réduit à l'état d'une poudre fine qui se mélange facilement au reste de la ration. Quant aux pommes de terre fraîches, conservées sur caillebotis pendant toute la durée de l'expérience, elles sont cuites à la vapeur au fur et à mesure des besoins à l'aide d'un cuiseur électrique.

\section{$1^{0}$ Expérience en lots $(A)$}

Trois lots de $\mathrm{I} 3$ porcs de race Large White, dont 6 mâles et 7 femelles, d'un poids moyen initial de $30 \mathrm{~kg}$ et provenant du troupeau expérimental de Jouy-en-Josas, sont constitués en tenant compte du sexe, de l'âge et du poids. Ils sont élevés en loges individuelles et nourris selon le système LEFMANN ; les quantités d'aliment concentré azoté et d'orge sont déduites d'expériences antérieures sur les betteraves à haute teneur en matière sèche (RÉRAT et HENRY, I964, I965a et $b$ ) : pratiquement on distribue chaque jour $300 \mathrm{~g}$ d'un aliment concentré azoté renfermant $44 \mathrm{p}$. Ioo de matières azotées totales et, suivant les lots:

- I : de l'orge volonté,

- II : I $200 \mathrm{~g}$ d'orge et des pommes de terre déshydratées à volonté, dès que les animaux ingèrent la totalité de leur ration concentrée.

- III : I $200 \mathrm{~g}$ d'orge et des pommes de terre fraîches à volonté dans les mêmes conditions que précédemment.

Le concentré azoté, l'orge et les pommes de terre sont administrés en 3 repas courts de 20 à 3 o minutes. Les aliments secs sont mélangés avant la distribution et humidifiés, à raison de 3 parties d'eau pour une partie d'aliment. Dans tous les cas, les animaux reçoivent de l'eau à volonté.

Au cours de la croissance, il est procédé à la pesée des animaux à intervalles réguliers (tous les I4 jours) et à l'enregistrement quotidien des quantités de nourriture consommées. Les animaux sont abattus au poids de $100 \mathrm{~kg}$; les modalités expérimentales concernant la découpe des carcasses et les critères retenus ont été décrits dans une publication antérieure (RÉRAT et HENRY, 1964).

\section{$2^{0}$ Expériences en cages de digestibilité $(B)$}

Six porcs mâles castrés, de race Large White et d'un poids moyen initial de $40,2 \mathrm{~kg}$, sont placés en cages de digestibilité et soumis, par groupe de 2 , à l'un des trois traitements précédents (I, II, III), suivant le dispositif du carré latin, afin d'estimer la valeur d'utilisation digestive des éléments énergétiques et azotés de chacun des trois aliments par la méthode de la différence (tabl. I).

$\mathrm{Au}$ cours d'une première période de I4 jours, les animaux reçoivent quotidiennement I $500 \mathrm{~g}$ d'un régime de base (RB) composé de $300 \mathrm{~g}$ de concentré azoté et $\mathrm{I} 200 \mathrm{~g}$ d'orge. Pendant les 3 périodes suivantes, de même durée que la précédente, on distribue, en plus du régime de base, une quantité équivalente de matière sèche de chacun des trois aliments: soit $800 \mathrm{~g}$ d'orge (traitement $\mathrm{I}$ ), $800 \mathrm{~g}$ de pommes de terre déshydratées (traitement II) ou l'équivalent en matière sèche de pommes de terre fraîchement cuites (traitement III). Une dernière période est consacrée au régime de base (RB) seul dans les mêmes conditions que la première, afin de contrôler l'évolution des critères d'utilisation digestive des éléments énergétiques et azotés du régime en fonction de l'âge ou du poids vif. La durée de chaque période (I4 jours) a été choisie de façon à aménager une période de transition de 8 jours suivie d'une période de collecte de 6 jours. 
La veille de chaque période, on procède à la pesée et à l'ensachage de la totalité des rations journalières correspondantes, tandis qu'un échantillon est prélevé en vue du contrôle de la teneur en matière sèche des différents aliments. Cependant, en ce qui concerne les pommes de terre cuites, qui sont distribuées à l'état frais, les pesées sont effectuées quotidiennement et la teneur en matière sèche est contrôlée dans les mêmes conditions que précédemment. Les rations journalières sont distribuées en trois repas humides, à raison de trois parties d'eau pour une partie d'aliment sec. I.es refus sont recueillis, stockés en chambre froide à $3-4^{\circ} \mathrm{C}$, puis séchés à $70^{\circ} \mathrm{C}$ dans un four à ventilation et pesés à l'issue de chaque période de collecte.

TABLEAU I

Expérience $B-$ Schéma expérimental

\begin{tabular}{|c|c|c|c|c|c|}
\hline \multirow{2}{*}{ Porcs $n^{0 s}$} & \multicolumn{5}{|c|}{ Périodes } \\
\hline & 1 & 2 & 3 & 4 & 5 \\
\hline $\begin{array}{l}1 \text { et } 2 \\
3 \text { et } 4 \\
5 \text { et } 6\end{array}$ & $\begin{array}{l}\text { RB } \\
\text { RB } \\
\text { RB }\end{array}$ & $\begin{array}{l}\text { I } \\
\text { II } \\
\text { III }\end{array}$ & $\begin{array}{l}\text { II } \\
\text { III } \\
\text { I }\end{array}$ & $\begin{array}{l}\text { III } \\
\text { I } \\
\text { II }\end{array}$ & $\begin{array}{l}\text { RB } \\
\text { RB } \\
\text { RB }\end{array}$ \\
\hline
\end{tabular}

Sur les échantillons représentatifs moyens du régime de base et de chacun des aliments on effectue les déterminations suivantes :

- matière sèche, par séchage à l'étuve à $105^{\circ} \mathrm{C}$ pour les aliments concentrés et à $70^{\circ} \mathrm{C}$ pour les tubercules frais;

- cendres, par incinération au four à $55^{\circ} \mathrm{C}$;

- azote, par la méthode Kjeldahl ;

- énergie, par combustion dans un calorimètre adiabatique "Gallenkamp ".

Les émissions fécales sont recueillies quotidiennement pendant les 6 jours de la période principale, pesées à l'état frais, puis stockées en récipient hermétique dans un congélateur à $-\mathrm{I} 5^{\circ} \mathrm{C}$. A l'issue de cette période, elles sont homogénéisées dans un mélangeur de marque "Stephan ". La teneur en matière sèche est déterminée sur deux prises aliquotes d'environ $200 \mathrm{~g}$ après séchage dans un four à ventilation à $70^{\circ} \mathrm{C}$. Une troisième prise aliquote d'environ $500 \mathrm{~g}$ est réhomogénéisée dans un mixer "Waring Blendor " après addition d'une quantité connue d'eau, la teneur en matière sèche du produit final étant de l'ordre de ro à $15 \mathrm{p}$. 1oo. Sur des prises aliquotes, on procède à l'analyse de la matière sèche, des cendres, de l'azote et de l'énergie, selon les méthodes exposées ci-dessus. Les animaux sont pesés une fois par semaine.

\section{RÉSULTATS}

\section{I. - EXPÉRIENCE A}

\section{Io Composition chimique des aliments et des régimes}

Les résultats moyens d'analyse des aliments et du concentré azoté sont rapportés dans le tableau 2. La teneur en matières azotées $(\mathrm{N} \times 6,25)$ relativement à la matière sèche est sensiblement la même dans le cas des pommes de terre déshydratées et fraîches après cuisson (respectivement $I \mathbf{I}, 7$ et $I I, 6$ p. Ioo) et peu différente de celle de l'orge (II,9 p. IOO). Le régime de base, composé de $300 \mathrm{~g}$ de concentré azoté et I $200 \mathrm{~g}$ d'orge, renferme ainsi I7,3 p. Ioo de matières azotées. Notons par ailleurs que le pourcentage de cendres est nettement plus élevé dans les pommes de terre 
déshydratées que dans les pommes de terre fraîches (plus de 9 p. Ioo contre 6 p. Ioo) ; cette différence vient du fait que les tubercules n'avaient pas été lavés avant leur déshydratation.

\section{'TABLEAU 2}

Composition chimique des aliments et des régimes

(Expérience A)

\begin{tabular}{|c|c|c|c|c|}
\hline Aliment & Concentré azoté (1) & Orge & $\begin{array}{l}\text { Pommes de terre } \\
\text { déshydratées }\end{array}$ & $\begin{array}{l}\text { Pommes de terre } \\
\text { fraîches après } \\
\text { cuisson }\end{array}$ \\
\hline $\begin{array}{c}\text { Matière sèche } \% \ldots \ldots \ldots \\
\text { Composition } \% \text { m. sèche }\end{array}$ & 92,33 & 89,67 & 91,55 & 24,80 \\
\hline Matières minérales (cendres). & 20,95 & 2,90 & 9,34 & 6,03 \\
\hline Matières grasses . . . . . . . & 1,98 & 1,98 & 0,25 & 0,36 \\
\hline Mat. azotées $(\mathrm{N} \times 6,25) \ldots$ & 47,89 & 11,94 & 11,67 & 11,59 \\
\hline Cellulose brute (Weende)... & 4,82 & 4,99 & 3,75 & 2,74 \\
\hline Extractif non azoté...... & 24,36 & 78,19 & 74,99 & 79,28 \\
\hline
\end{tabular}

(1) Composition p. 100 : tourteau de soja, 22,5; tourteau d'arachide, 22,5; farine de viande, 22,5; farine de poisson du Pérou, 12,5; levure de distillerie, 10 ; mélange minéral et vitaminique, 10. La composition du mélange minéral est la suivante en p. 100 : phosphate bicalcique, 41,5, carbonate de calcium, 22,5 ; carbonate de magnésium léger, 10,0 ; chlorure de sodium, 24,0 ; sulfate ferreux $\left(7 \mathrm{H}_{2} \mathrm{O}\right): 1,0$; sulfate de manganèse, $\mathrm{H}_{2} \mathrm{O}, 0,30$; sulfate de cuivre, $5 \mathrm{H}_{2} \mathrm{O}, 0,10$; carbonate de zinc, 0,50 ; sulfate de cobalt, $7 \mathrm{H}_{2} \mathrm{O}, 0,05$; iodure de potassium, 0,05. La composition du mélange vitaminique a été rapportée par ailleurs (RÉrat et HENRY, 1964).

\section{$2^{\circ}$ Croissance et consommation}

Les résultats généraux de croissance et de consommation sont présentés dans les tableaux 3, 4 et 5 , respectivement pour la période totale $30-\mathrm{I} 00 \mathrm{~kg}$ de poids vif et pour les périodes $30-60 \mathrm{~kg}$ et $60-\mathrm{I} 00 \mathrm{~kg}$.

a) Vitesse de croissance.

Les animaux recevant de l'orge (lot I) présentent une vitesse de croissance significativement plus élevée que ceux nourris avec des pommes de terre (lots II et III), mais la différence entre ces deux derniers lots n'est pas significative. L'administration de pommes de terre déshydratées et fraîches se traduit par un allongement de la durée totale d'engraissement de I3 et 9 jours respectivement, correspondant à une réduction de la vitesse de croissance de $\mathrm{r}_{5}$ et $\mathrm{I}_{2} \mathrm{p}$. Ioo.

Les écarts de croissance enregistrés sont surtout importants pendant la période de finition (6o-roo $\mathrm{kg}$ de poids vif), comme le montre d'ailleurs l'évolution du gain de poids cumulé (fig. I) ; ceci s'explique par le fait que la part des pommes de terre dans l'ingestion totale est alors plus élevée.

\section{b) Consommation de nourriture.}

Pour passer de 30 à roo $\mathrm{kg}$ de poids vif, les animaux ont consommé Io $8 \mathrm{~kg}$ de poudre de pommes de terre ou $336 \mathrm{~kg}$ de pommes de terre fraîches ; les consomma- 
tions correspondantes de matière sèche représentent respectivement 40 et $37 \mathrm{p}$. roo de la consommation totale de matière sèche.

La consommation journalière de matière sèche est significativement plus élevée dans le lot " orge" (I), que dans les lots " pommes de terre" (II et III) soit 2,24 kg contre 2,07 et $1,98 \mathrm{~kg}$. La comparaison de ces deux derniers lots fait ainsi apparaître un accroissement de la consommation de matière sèche chez les animaux recevant des pommes de terre déshydratées, la différence n'étant toutefois pas significative. Par ailleurs, il faut noter que la distribution de poudre de pommes de terre a dû être limitée dans certains cas, par suite d'accès de diarrhée chez les animaux.

\section{TABLEAU 3}

Résultats généraux entre 30 et $100 \mathrm{~kg}$ de poids vif

(Expérience A)

\begin{tabular}{|c|c|c|c|c|}
\hline Lot $\ldots \ldots \ldots \ldots \ldots \ldots \ldots$ & I & II & III & \\
\hline Aliment..$\ldots \ldots \ldots \ldots \ldots$ & Orge & $\begin{array}{l}\text { Pommes de terre } \\
\text { déshydratées }\end{array}$ & $\begin{array}{l}\text { Pommes de terre } \\
\text { cuites }\end{array}$ & $\begin{array}{l}\text { Écart-type de la } \\
\text { moyenne } s_{x}^{-\left({ }^{2}\right)}\end{array}$ \\
\hline $\begin{array}{l}\text { Poids initial }(\mathrm{kg}) \ldots \ldots \ldots \ldots \\
\text { Poids final }(\mathrm{kg}) \ldots \ldots \ldots \ldots \ldots \\
\text { Durée (jours) } \ldots \ldots \ldots \ldots \ldots \ldots \\
\text { Gain moy./j }(\mathrm{g}) \ldots \ldots \ldots \ldots \ldots\end{array}$ & $\begin{array}{r}30,2 \\
99,8 \\
104,5 \\
672\end{array}$ & $\begin{array}{r}32,2 \\
98,8 \\
117,9 \\
568 \\
\end{array}$ & $\begin{array}{r}32,4 \\
99,4 \\
113,6 \\
592 \\
\end{array}$ & 15,5 \\
\hline $\begin{array}{l}\text { Consommation totale }(\mathrm{kg}) \\
\quad \text { Matière fraîche } \\
\text { Aliment concentré } \ldots \ldots \ldots \ldots \\
\text { Pommes de terre } \ldots \ldots \ldots \ldots \ldots\end{array}$ & 259,1 & $\begin{array}{l}161,1 \\
108,1\end{array}$ & $\begin{array}{l}156,5 \\
336,2\end{array}$ & \\
\hline $\begin{array}{l}\text { Matière sèche: } \\
\text { Aliment concentré } \ldots \ldots \ldots \ldots \\
\text { Pommes de terre } \ldots \ldots \ldots \ldots \ldots \\
\text { Totale } \ldots \ldots \ldots \ldots \ldots \ldots \ldots\end{array}$ & $\begin{array}{r}233,1 \\
- \\
233,1\end{array}$ & $\begin{array}{r}145,8 \\
98,9 \\
24 x, 5\end{array}$ & $\begin{array}{r}141,6 \\
82,7 \\
224,3\end{array}$ & \\
\hline $\begin{array}{l}\text { Consommation journalière }(\mathrm{kg}) \\
\quad \text { Matière fraîche : } \\
\text { Aliment concentré........... } \\
\text { Pommes de terre........... }\end{array}$ & $2,50\left({ }^{3}\right)$ & $\begin{array}{l}1,37 \\
0,92\end{array}$ & $\begin{array}{l}1,38 \\
2,97\end{array}$ & \\
\hline $\begin{array}{l}\text { Matière sèche : } \\
\text { Aliment concentré.......... } \\
\text { Pommes de terre } \ldots \ldots \ldots \ldots \ldots \\
\text { Totale } \ldots \ldots \ldots \ldots \ldots \ldots \ldots\end{array}$ & $\begin{array}{l}2,24\left(^{4}\right) \\
2,2 !\end{array}$ & $\begin{array}{l}1,2: 3 \\
0,8 t \\
2,07\end{array}$ & $\begin{array}{l}1,25 \\
0,73 \\
1,98 \\
\end{array}$ & 0,039 \\
\hline 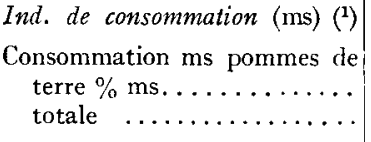 & $\begin{array}{l}3,35 \\
-\end{array}$ & $\begin{array}{c}3,67 \\
40,1\end{array}$ & $\begin{array}{c}3,35 \\
36,9\end{array}$ & 0,057 \\
\hline
\end{tabular}

(1) Quantité de matière sèche consommée $(\mathrm{kg}) / \mathrm{gain}$ de poids $(\mathrm{kg})$.

(2) Parmi les moyennes analysées statistiquement seules sont différentes au seuil 0,05 celles qui ne sont pas reliées par un trait (test de KEuLs, cité par SNEDECoR, 1956).

(3) dont 0,27 de concentré azoté.

$\left({ }^{4}\right)$ dont 0,25 de concentré azoté. 
L'évolution de la consommation journalière de pommes de terre déshydratées et fraîches est représentée graphiquement dans les figures 2 et 3 . La quantité de pommes de terre déshydratées augmente progressivement de $200 \mathrm{~g}$ par jour à $35 \mathrm{~kg}$ de poids vif à plus de $I, 500 \mathrm{~kg}$ à $90 \mathrm{~kg}$. La quantité de pommes de terre fraîches augmente de la même façon de $0,7 \mathrm{~kg}$ à $35 \mathrm{~kg}$ de poids vif à $5,2 \mathrm{~kg}$ à $90 \mathrm{~kg}$; en pourcentage du poids, elle augmente rapidement à partir de 2 p. Ioo à $35 \mathrm{~kg}$ pour plafonner aux environs de 5 à $6 \mathrm{p}$. Ioo au-delà de $60 \mathrm{~kg}$ de poids vif.

Si l'on considère l'évolution de la quantité journalière de matière sèche ingérée, on constate que les écarts de consommation entre les lots " orge " et " pommes de terre " sont d'autant plus importants que le poids vif augmente (fig. $4 a$ ) ; en outre, la consommation de matière sèche de pommes de terre est plus faible à l'état frais

TABLEAU 4

Résultats entre 30 et $60 \mathrm{~kg}$ de poids vif

(Expérience A)

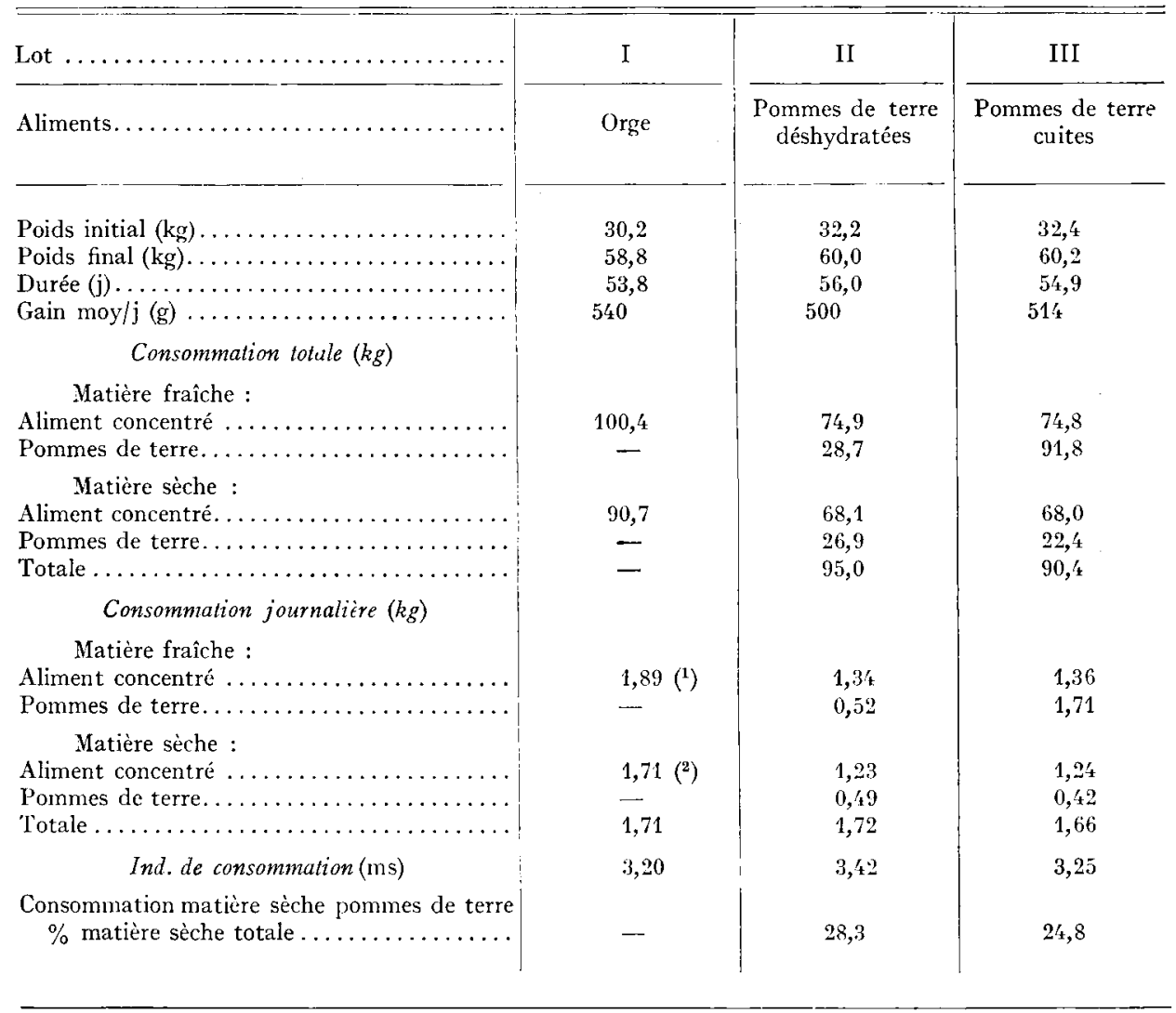

(1) dont 0,26 de concentré azoté.

(2) dont 0,24 de concentré azoté. 
que sous forme déshydratée. Les mêmes différences apparaissent si 1'on exprime la quantité de matière sèche ingérée en pourcentage du poids vif (fig. $4 b$ ); alors que dans le lot "orge " elle se situe pratiquement aux environs de $4 \mathrm{p}$. Ioo, dans le lot " pommes de terre ", elle subit une diminution sensible entre 35 et $90 \mathrm{~kg}$, passant ainsi respectivement de plus de $4 \mathrm{p}$. Ioo à moins de $3 \mathrm{p}$. Ioo du poids vif.

En définitive, la part des pommes de terre dans la consommation totale de matière sèche croit à mesure que les animaux augmentent de poids, d'un peu plus de Io p. IOo à $35 \mathrm{~kg}$ à plus de $50 \mathrm{p}$. IOO aux alentours de Ioo $\mathrm{kg}$ (fig. 5) ; de plus, elle est légèrement plus élevée lorsque les pommes de terre sont administrées sous forme déshydratée.

c) Indice de consommation.

Afin de comparer les aliments entre eux, l'indice de consommation a été exprimé

TABLEAU 5

Résultats entre 60 et $100 \mathrm{~kg}$ de poids vif

(Expérience A)

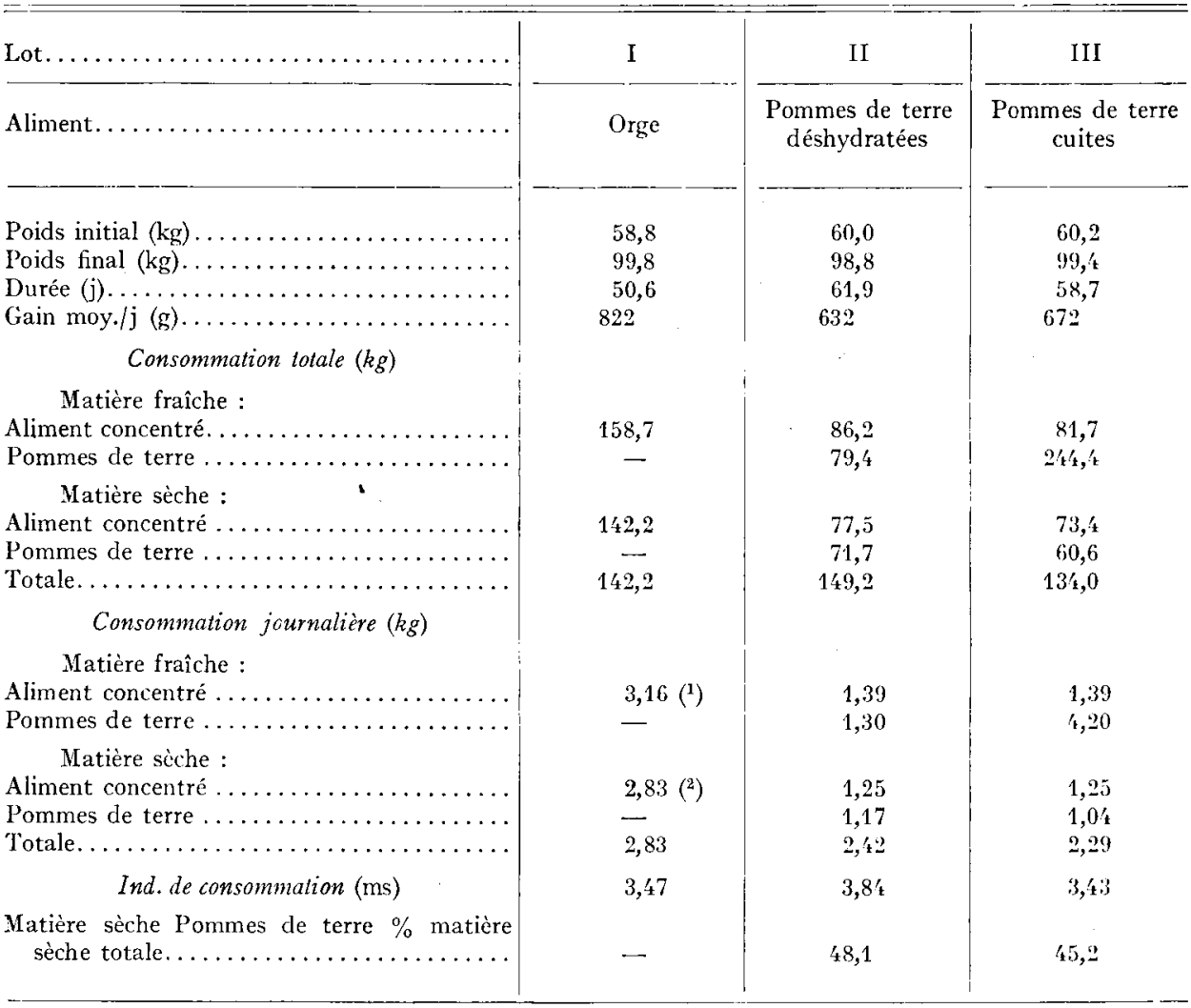

(1) dont 0,28 de concentré azoté.

( $\left.{ }^{2}\right)$ dont 0,26 de concentré azoté. 
en matière sèche consommée $(\mathrm{kg})$ par unité de gain de poids vif $(\mathrm{kg})$. Les valeurs ainsi obtenues ne font apparaître aucune différence entre les lots " orge » et " pommes de terre fraîches ", qu'il s'agisse de la période totale ou des périodes $30-60 \mathrm{~kg}$ et

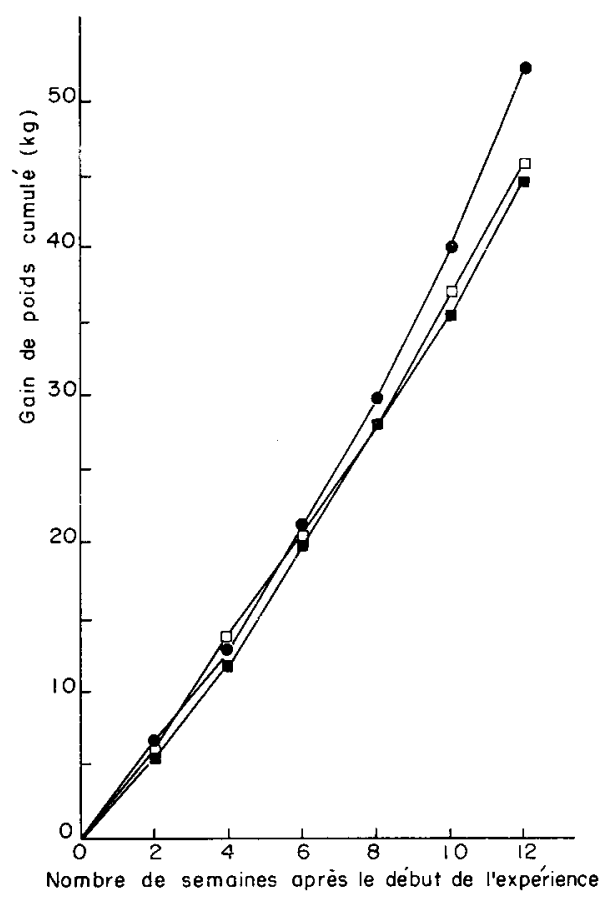

Fig. I. - Évolution du gain de poids cumulé

orge; $\longrightarrow$ PdT déshydratées; $\square \longrightarrow \square$ PdT fraîchee.

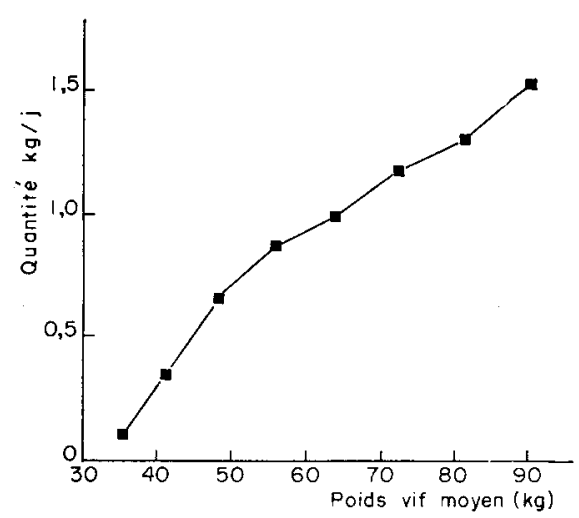

FIG. 2. - Consommation journalière de pommes de terre déshydratées en fonction du poids vif

60-I00 kg de poids vif ; par contre, l'efficacité globale de la ration est significativement plus faible dans le lot " pommes de terre déshydratées », l'indice de consommation étant de 3,67 contre 3,35 dans les deux autres lots entre 30 et Ioo $\mathrm{kg}$ de poids vif. 

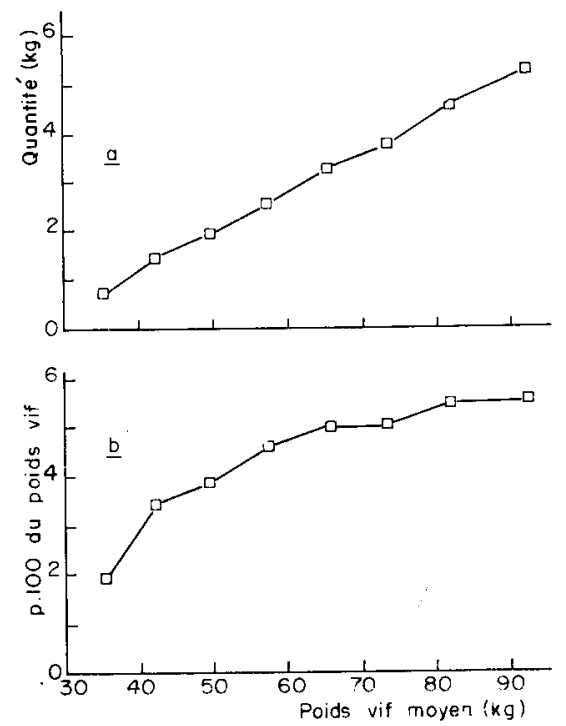

Fig. 3. - Consommation journalière de pommes de lerre frầches en fonction du poids vif

a) en quantité absolue, $\mathrm{kg} / \mathrm{j}$;

b) en pourcentage du poids vif.
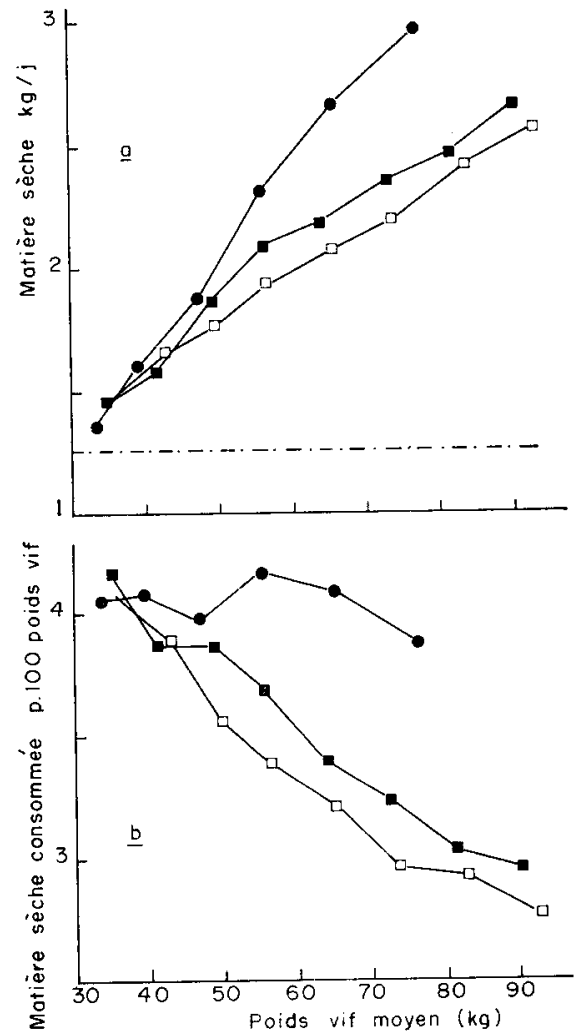

FIc. 4. - Consommation journalière de matière sèche en fonction du poids vif

a) en quantité absolue, $\mathrm{kg} / \mathrm{j}$;

$b$ ) en pourcentage du poids vif.

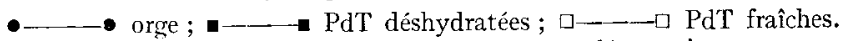
-...- aliment concentré complémentaire 
A partir des résultats précédents et connaissant les parts respectives des pommes de terre et de l'orge dans la consommation totale de matière sèche, il est possible de calculer la valeur de remplacement des pommes de terre déshydratées et fraîches vis-à-vis de l'orge, pris comme aliment de référence, selon la méthode de substitution (ARMSBY, I922), utilisée antérieurement avec la betterave (RÉRAT et HENRY, I964). Bien entendu, ce mode d'estimation n'est exact que dans la mesure où la part de l'entretien dans le besoin global est la même pour les trois lots, ce qui n'est pas le cas en raison des différences dans l'intensité de croissance. Compte tenu de cette restriction, nous pouvons procéder à une estimation approchée de la valeur de rem-

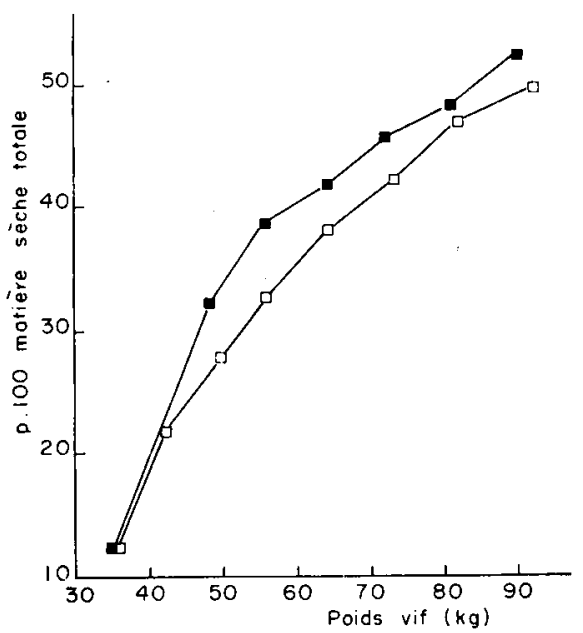

FiG. 5. - Consommation de pommes de terre en pourcentage de la matière sèche totale $\square$ PdT déshydratées;
$\square-\square \operatorname{PdT}$ fraîches.

placement à partir des quantités d'aliments nécessaires pour fournir un kilogramme de gain de poids; le détail du calcul est décrit dans le tableau 5 . Les quantités de pommes de terre déshydratées et fraîches équivalentes à I $\mathrm{kg}$ d'orge (ou UF) sont respectivement $\mathrm{I}, 25$ et $3,64 \mathrm{~kg}$. Les valeurs fourragères correspondantes sont respectivement 0,80 et 0,27 UF par kg d'aliment frais, ou 0,87 et I, Io UF par $\mathrm{kg}$ de matièrè sèche. En définitive, la déshydratation de la pomme de terre entraîne une diminution de la valeur fourragère d'environ 20 p. I00, ce qui peut être attribué à plusieurs causes dont il est fait état dans la discussion.

\section{$3^{\circ}$ Composition corporelle}

L'examen du tableau 7 fait ressortir des différences importantes au niveau de la composition corporelle selon le type d'aliment utilisé. Le rendement des carcasses est significativement plus élevé dans le lot " orge " que dans les lots " pommes de terre ". Le pourcentage de morceaux nobles (jambon + longe) dans la carcasse 


\section{TABLEAU 6}

Valeur de remplacement de la pomme de terre par rapport à l'orge (Expérience A)

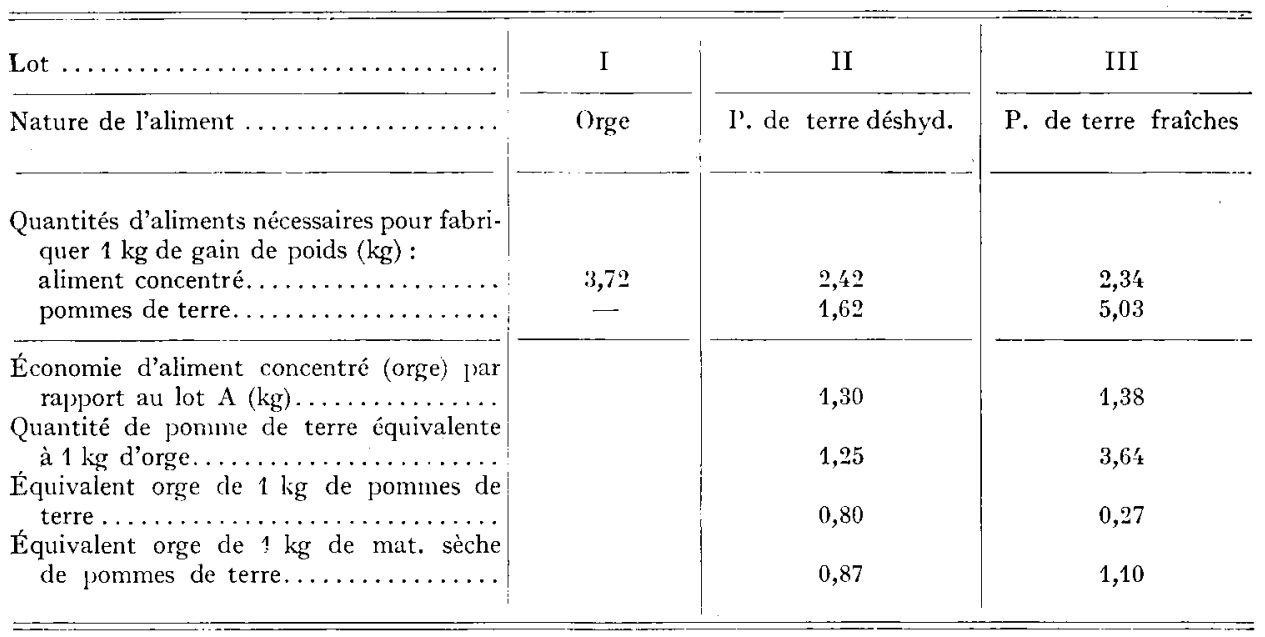

\section{TABLEAU 7}

Résultats concernant la composition corporelle

(Expérience A)

\begin{tabular}{|c|c|c|c|c|}
\hline Lot $\ldots \ldots \ldots \ldots \ldots \ldots \ldots \ldots \ldots \ldots \ldots \ldots \ldots$ & I & II & III & \\
\hline Aliment..$\ldots \ldots \ldots \ldots \ldots \ldots \ldots \ldots$ & Orge & $\begin{array}{c}\text { Pommes de } \\
\text { terre } \\
\text { déshydratées }\end{array}$ & $\begin{array}{l}\text { Pommes de } \\
\text { terre } \\
\text { cuites }\end{array}$ & $\begin{array}{l}\text { Ecart-type de } \\
\text { la moyenne } s_{x}\end{array}$ \\
\hline $\begin{array}{l}\text { Poids vif final }(\mathrm{kg}) \ldots \ldots \ldots \ldots \ldots \ldots \ldots \\
\text { Poids net }(\mathrm{kg}) \ldots \ldots \ldots \ldots \ldots \ldots \ldots \\
\text { Rendement }: \text { poids vif } \% \text { poids net } \ldots \ldots\end{array}$ & $\begin{array}{l}99,8 \\
72,2 \\
72,26\end{array}$ & $\begin{array}{l}98,8 \\
70,1 \\
70,93 \\
\end{array}$ & $\begin{array}{l}99,4 \\
69,9 \\
70,29\end{array}$ & 0,42 \\
\hline $\begin{array}{l}\text { Jambon } \% \text { poids net } \ldots \ldots \ldots \ldots \ldots \ldots \ldots \\
\text { Longe } \% \text { poids net } \ldots \ldots \ldots \ldots \ldots \ldots \ldots \\
\text { (Jambon }+ \text { Longe) } \% \text { poids net } \ldots \ldots \ldots\end{array}$ & $\begin{array}{l}21,13 \\
27,23 \\
48,68 \\
\end{array}$ & $\begin{array}{l}22,06 \\
27,91 \\
50,01 \\
\end{array}$ & $\begin{array}{l}22,54 \\
29,85 \\
52,39\end{array}$ & 0,58 \\
\hline (Bardière + Panne) $\%$ poids net..... & 19,82 & 19,32 & 17,10 & 0,70 \\
\hline$E_{p} . \operatorname{lard}\left(\frac{\text { Rein }+ \text { Dos }}{2}\right), m m \ldots \ldots \ldots$ & 30.7 & 28,9 & 26,2 & 1,4 \\
\hline Longueur, $\mathrm{cm} . \ldots \ldots \ldots \ldots \ldots \ldots$ & 95,7 & 96,0 & 95,8 & \\
\hline
\end{tabular}

Seules les moyennes non réunies par un trait sont significativement différentes au seuil 0,05 (test de KEULS, cité par SNEDECOR, 1956). 
est significativement plus élevé chez les animaux recevant des pommes de terre fraîches que chez ceux recevant de l'orge ou des pommes de terre séchées ; inversement, le pourcentage de morceaux gras (bardière + panne) dans la carcasse est plus faible. La même tendance se manifeste au niveau de l'épaisseur du lard dorsal; cependant la grande variabilité de ce critère ne permet pas de déceler un écart significatif.

\section{II. - EXXṔRIENCE B}

Les résultats généraux de digestibilité sont présentés dans le tableau 9. Ils concernent quatre animaux seulement sur les six initialement prévus dans le protocole. En effet deux porcs ont été atteints de diarrhées intermittentes, de sorte qu'il n'a pas été possible dans ce cas de procéder à une collecte quantitative des fèces.

TABLEAU 8

Résultats d'analyse du régime de base et des aliments

(Expérience B)

\begin{tabular}{|c|c|c|c|c|}
\hline & $\begin{array}{l}\text { Régime de } \\
\text { base (RB) }\end{array}$ & Orge & $\begin{array}{l}\text { Pommes de } \\
\text { terre séchées }\end{array}$ & $\begin{array}{l}\text { Pommes de } \\
\text { terre fraîche- } \\
\text { ment cuites }\end{array}$ \\
\hline Matière sèche $\% \ldots \ldots \ldots \ldots \ldots \ldots$ & 87,60 & 87,34 & 88,23 & 24,40 \\
\hline Matière organique $\%$ matière sèche..... & 93,76 & 97,05 & 91,05 & 95,11 \\
\hline $\mathrm{N} \%$ matière sèche $\ldots \ldots \ldots \ldots \ldots \ldots$ & 3,13 & 2,00 & 1,98 & 1,70 \\
\hline Énergie brute, kcal/g matière sèche..... & 4496 & 4572 & 4145 & 4363 \\
\hline
\end{tabular}

A partir des résultats des périodes I et 5 , nous avons ainsi calculé les coefficients d'utilisation digestive apparents (C. U. D.) des éléments énergétiques et azotés du régime de base (tabl. Io). Les valeurs trouvées pour les C. U. D. de la matière sèche, de la matière organique et de l'énergie sont pratiquement les mêmes au début et à la fin de l'expérience, alors que le poids vif moyen des animaux augmente de 40 à $70 \mathrm{~kg}$; les moyennes correspondantes sont respectivement 82,4, 84,7 et 83, I pour la matière sèche, la matière organique et l'énergie, tandis que la valeur énergétique du régime de base, exprimée en énergie digestible, est de $3739 \pm 9,5 \mathrm{kcal} / \mathrm{kg}$ de matière sèche. Par contre, si l'on considère les matières azotées, on constate que le C. U. D. apparent a tendance à augmenter lorsque le poids vif s'élève de 40 à $70 \mathrm{~kg}$, les valeurs moyennes étant respectivement $8 \mathrm{I}$, I et 83,4 .

Connaissant les coefficients d'utilisation digestive du régime de base, il est possible d'estimer, par la méthode de la différence, les coefficients correspondants des aliments (orge, pommes de terre déshydratées et pommes de terre fraîches). Les valeurs ainsi calculées sont rapportées dans le tableau II. Comme on peut le constater, les pommes de terre fraîches présentent une digestibilité de la matière sèche et de l'énergie significativement plus élevée que les pommes de terre déshydratées et 


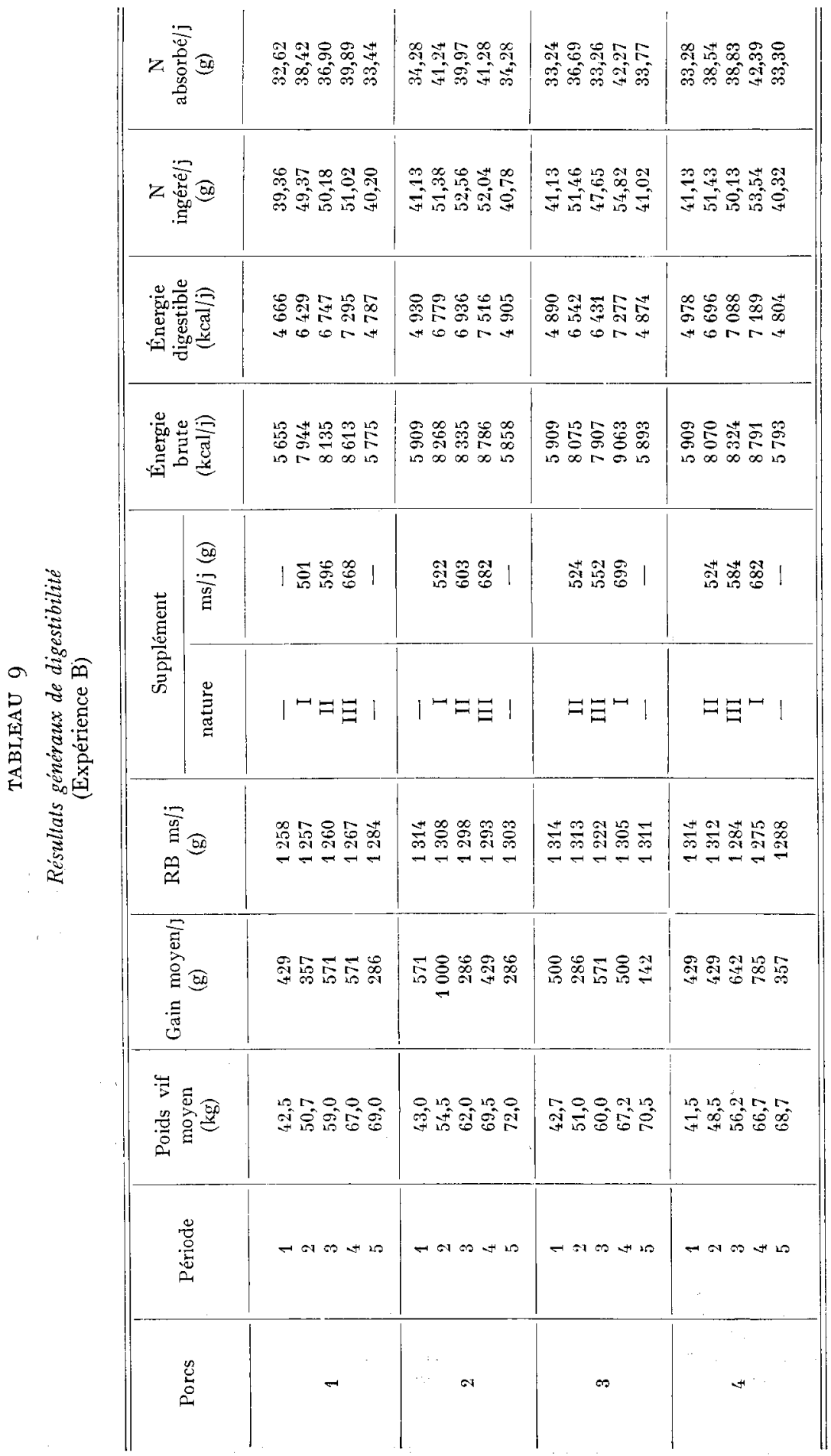


TABLEAU IO

Coefficient de digestibilité du régime de base

Expérience B

\begin{tabular}{|c|c|c|c|c|c|c|}
\hline Porc & Période & Matière sèche & Matière org. & Énergie & Azote & $\begin{array}{c}\text { Energie } \\
\text { digestible } \\
\text { (kcal/kg m. s.) }\end{array}$ \\
\hline 1 & $\begin{array}{l}1 \\
5\end{array}$ & $\begin{array}{l}82,0 \\
81,8\end{array}$ & $\begin{array}{l}84,3 \\
84,3\end{array}$ & $\begin{array}{l}82,5 \\
82,9\end{array}$ & $\begin{array}{l}82,9 \\
84,3\end{array}$ & $\begin{array}{l}3710 \\
3727\end{array}$ \\
\hline 2 & $\begin{array}{l}1 \\
5\end{array}$ & $\begin{array}{l}82,9 \\
82,7\end{array}$ & $\begin{array}{l}84,8 \\
84,9\end{array}$ & $\begin{array}{l}83,4 \\
83,7\end{array}$ & $\begin{array}{l}83,8 \\
84,1\end{array}$ & $\begin{array}{l}3752 \\
3765\end{array}$ \\
\hline 3 & $\begin{array}{l}1 \\
5\end{array}$ & $\begin{array}{l}82,1 \\
81,7\end{array}$ & $\begin{array}{l}81,3 \\
85,0\end{array}$ & $\begin{array}{l}82,8 \\
82,7\end{array}$ & $\begin{array}{l}80.8 \\
82,3\end{array}$ & $\begin{array}{l}3: 291 \\
3719\end{array}$ \\
\hline 4 & $\begin{array}{l}1 \\
5\end{array}$ & $\begin{array}{l}83,1 \\
82,1\end{array}$ & $\begin{array}{l}85,8 \\
84,5\end{array}$ & $\begin{array}{l}84,2 \\
82,9\end{array}$ & $\begin{array}{l}80,9 \\
82,6\end{array}$ & $\begin{array}{l}3788 \\
3729\end{array}$ \\
\hline \multicolumn{2}{|c|}{ Moyenne } & $\begin{array}{c}82,4 \\
\pm \quad 0,95(2)\end{array}$ & $\begin{array}{r}84,7 \\
-\quad 0,18\end{array}$ & $\begin{array}{c}83,1 \\
+\quad 0,20\end{array}$ & $\begin{array}{c}82,7(1) \\
\pm 0,18\end{array}$ & $\begin{array}{r}3739 \\
+\quad 9,5\end{array}$ \\
\hline
\end{tabular}

( ${ }^{1}$ Moyenne pour la période $1: 82,1 \pm 0,74$ et pour la période $5: 83,3 \pm 0,51$.

(2) Écart-type de la moyenne.

TABLEAU I I

Ligestibilité comparée de l'orge et de la pomme de terre

(Expérience B)

\begin{tabular}{|c|c|c|c|c|c|}
\hline & \multirow[b]{2}{*}{ Orge (I) } & \multirow{2}{*}{$\begin{array}{l}\text { Pommes de } \\
\text { terre déshy- } \\
\text { dratées (II) }\end{array}$} & \multirow{2}{*}{$\begin{array}{l}\text { Pommes de } \\
\text { terre fraîches } \\
\quad \text { (III) }\end{array}$} & \multicolumn{2}{|c|}{$\begin{array}{l}\text { Signification } \\
\text { statistique }\end{array}$} \\
\hline & & & & $\left.s_{x}{ }^{1}\right)$ & $\begin{array}{l}\text { Comparaison } \\
\text { des moyennes } \\
(\mathbf{2})\end{array}$ \\
\hline CUD matière sèche $\ldots \ldots \ldots \ldots \ldots$ & $78,9 \pm 1,11$ & $81,5 \pm 1,10$ & $87,2 \pm 2,40$ & 0,87 & III II I \\
\hline CUD matière organique .......... & $79,2 \pm 1,70$ & $8:, 6^{\prime} \pm 1,52$ & $87,4 \pm 2,40$ & 0,93 & III $\longdiv { \text { II I } }$ \\
\hline CUD énergie $\ldots \ldots \ldots \ldots \ldots \ldots$ & $77,2 \pm 0,62$ & $80,9 \pm 1,56$ & $85,4 \pm 2,56$ & 1,16 & III II I \\
\hline CUD azoté $\ldots \ldots \ldots \ldots \ldots \ldots \ldots$ & $65,3 \pm 1,59$ & $35,4 \pm 6,35$ & $49,8 \pm 9,93$ & 6,10 & I III $\overline{\text { II }}$ \\
\hline 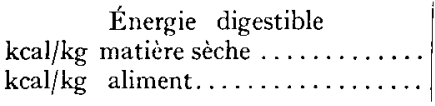 & $\begin{array}{l}3531 \\
308^{\prime}\end{array}$ & $\begin{array}{l}3352 \pm 65 \\
2957\end{array}$ & $\begin{array}{c}3761 \\
918\end{array}$ & 51 & III I II \\
\hline
\end{tabular}

(1) $s_{x}^{-}:$Écart-type de la moyenne au seuil 0,05 .

(2) Seules les moyennes non réunies par un trait sont significativement différentes au seuil 0,05 (test de KEULS, cité par SNEDECOR, 1956).

Nota. Les CUD de la matière sèche, de la matière organique et de l'énergie ont été calculés à partir de la valeur moyenne du CUD correspondant du Régime de Base. En ce qui concerne les matières azotées, e même calcul a été effectué à partir du CUD de la période adjacente pour les périodes extrêmes et à partir du CUD moyen pour la période intermédiaire. 
l'orge, l'écart entre ces deux derniers aliments n'étant pas significatif. Mais si l'on considère la digestibilité de la matière organique, les pommes de terre fraîches et déshydratées se révèlent supérieures à l'orge et ne sont pas significativement différentes. Les valeurs énergétiques, exprimées en énergie digestible par $\mathrm{kg}$ de matière sèche, sont respectivement $353 \mathrm{r}, 335^{2}$ et $376 \mathrm{r} \mathrm{kcal}$ pour l'orge, les pommes de terre déshydratées et les pommes de terre fraîches, soit par kg d'aliment 3 o84, 2957 et $9 \mathrm{I} 8 \mathrm{kcal}$ digestibles. Par ailleurs, la comparaison des C. U. D. des matières azotées fait ressortir des différences importantes d'un aliment à l'autre; malgré la grande variabilité de ce critère, la valeur la plus élevée est obtenue pour l'orge $(65,3)$ et la plus faible pour les pommes de terre déshydratées $(35,4$ conre 49,8 pour les tubercules frais).

\section{NABIEAU I2}

Consommations myyennes journalières d'énergie et de matières azotées digestibles

\begin{tabular}{|c|c|c|c|}
\hline Lot $\ldots \ldots \ldots \ldots \ldots \ldots \ldots \ldots \ldots \ldots \ldots$ & $\mathrm{I}$ & II & III \\
\hline Aliments . . . . . . . . . . . . . . . & Orge & Pd'T déshydratées & PdT fraîches \\
\hline $\begin{array}{l}\text { Matières azotées digestibles }(g / j) \ldots \ldots \ldots \ldots \\
\text { Énergie, (kcal dig. } . j) \ldots \ldots \ldots \\
\text { M. azot. dig. (g/1 } \ldots 00 \mathrm{kcal} \text { dig.) } \ldots \ldots \ldots \ldots \ldots\end{array}$ & $\begin{array}{r}274 \\
8163 \\
33,6\end{array}$ & $\begin{array}{r}2,31 \\
7415 \\
31,2\end{array}$ & $\begin{array}{r}2+2 \\
7419 \\
\quad 32.6\end{array}$ \\
\hline
\end{tabular}

A partir des coefficients de digestibilité des éléments énergétiques et azotés, nous avons calculé, pour les trois lots de l'expérience $A$, les consommations moyennes journalières de matières azotées digestibles et d'énergie digestible (tab1. I2) ; on peut remarquer que les consommations des lots "pommes de terre " sont nettement plus faibles que celles du lot " orge ». De plus, la comparaison des lots " orge " et "pommes de terre déshydratées " fait apparaître dans ce dernier un déficit relatif de matières azotées par rapport à l'énergie : 2,4 g de matières azotées digestibles pour I ooo kcal digestibles, soit $\mathrm{I} 8 \mathrm{~g}$ pour la ration moyenne journalière. Ce déficit peut être compensé par un apport complémentaire de $350 \mathrm{~g}$ de concentré azoté, au lieu de $300 \mathrm{~g}$, ce qui revient à élever le taux de matières azotées totales dans les $\mathrm{I} 500 \mathrm{~g}$ du régime de base de I7,5 à I8,5 p. Ioo.

\section{DISCUSSION}

L'introduction de pommes de terre cuites dans l'alimentation du porc en croissance provoque une diminution sensible de la consommation journalière de matière sèche, et du gain de poids vif, par référence à un régime exclusivement concentré. L,es résultats ainsi obtenus confirment les observations antérieurement formulées à ce sujet par LEROY, ZELTER et FÉVRIER (I952). Comme l'ont souligné les auteurs précédents, la différence entre l'utilisation de l'orge et celle des pommes de terre 
cuites provient certainement de la plus grande lenteur avec laquelle les animaux consomment les tubercules. Cette lenteur devient, lorsque l'animal dépasse 50 à $60 \mathrm{~kg}$ de poids vif, une cause de diminution de la consommation, ce qui explique la réduction comparée du gain de poids; en outre la dépense énergétique liée au repas augmente (LEROX, 1965). Le même phénomène a été observé par ailleurs dan: le cas de la betterave (RÉRAT et HENRY, I964). Pour pallier ce déficit énergétique, i1 est nécessaire de prévoir un apport suffisant d'aliment concentré complémentaire. Or, d'après un certain nombre d'auteurs (PIEPER, I962; SEEFEL.DT, I962; ECKHOFF, I963; FENDER et al., I963; LANTzsch et al., I964; Hoser, I965), le niveau de complémentation préconisé dans la méthode LEHMANn classique (LEHMANn, I929), soit I $\mathrm{kg}$ d'aliment concentré, s'est révélé insuffisant pour obtenir une croissance satisfaisante, la quantité généralement recommandée étant de I,50o kg. C'est précisément ce niveau de complémentation qui a été choisi dans la présente étude ; il paraît cependant insuffisant puisque les performances de croissance ne sont pas maxima. Quoi qu'il en soit, les pommes de terre fraîches présentent une valeur fourragère relativement élevée, de l'ordre de I,I UF par $\mathrm{kg}$ de matière sèche, et comparable à celle trouvée antérieurement par LEROY, ZELTER et FÉVRIER (I952). Étant donné par ailleurs que les matières azotées de la pomme de terre sont mieux équilibrées que celles de 1'orge en certains aminoacides indispensables, en particulier la lysine (Mangold et Columbus, i938; Hutchinson et al., I943; KAstel, , i957 ; HuGHES, I958; SchupHAN, I959), on peut s'attendre à ce que les carcasses obtenues soient plus maigres, en raison précisément d'un meilleur équilibre entre l'énergie et les aminoacides indispensables. La diminution du rendement des carcasses, consécutive à l'ingestion de pommes de terre, est également un phénomène connu, par suite d'un développement plus important du tractus digestif (LEROY, ZELTER et FÉvrier, I952; Richter et $a l$., I956 ; Weniger, I956). Notons d'ailleurs que cette diminution du rendement se retrouve avec le produit séché ; la même constatation a été faite par FRIEND et al. (I963) dans le cas de la pulpe de pomme de terre séchée.

Si l'on examine les résultats de digestibilité des matières azotées, on est frappé par la faiblesse des valeurs observées, en comparaison avec celles qu'on peut trouver dans la littérature ; il en est ainsi aussi bien de l'orge que des pommes de terre fraîches ou déshydratées. Il est possible que ces écarts systématiques soient dus à la méthode de calcul employée. Ainsi, le C.U.D des matières azotées du régime de base, qui est composé essentiellement d'orge, est nettement plus élevé que celui de l'orge ellemême $(82,7$ contre 65,3$)$; le même écart apparaît dans les résultats de NEHRING et al. (Ig60) qui, utilisant la même méthode par différence trouvent pour l'orge un C.U.D des matières azotées de $7 \mathrm{I}, 2 \mathrm{p}$. Ioo contre 82,3 pour le régime de base, ce dernier renfermant 63 p. Ioo d'orge et 23 p. Ioo d'avoine. On peut dès lors conclure à une surestimation du C.U.D azoté du régime de base, ce qui expliquerait les faibles valeurs obtenues par différence.

L'influence de la déshydratation sur la valeur nutritive de la pomme de terre a fait l'objet d'un grand nombre de travaux dont les premiers, réalisés par KELLNER, remontent à I908 (KELLNER et al., I908; KELLNER et NEUMANN, I9Io). Ein réalité, les résultats obtenus sont plus ou moins variables selon la nature du traitement appliqué. Ainsi, deux modes de déshydratation ont été utilisés, suivant que les tubercules sont étuvés, puis séchés sur cylindre à l'état de flocons, ou simplement pressés à l'état cru pour éliminer l'eau, puis séchés à l'état de cossettes ; ces traitements ont 
été décrits en détail par Fingerling (I933), STAHL et Friedrich (I940), Woodman et EVANS (I943), WOHLBIER et NECKERMANN (I952). D'une manière générale, il a été observé que les pommes de terre séchées en flocons présentent une valeur nutritive élevée, comparable à celle des tubeicules frais après cuisson ou à celle des céréales les plus énergétiques (maïs), et supérieure à celle des cossettes séchées. C'est ce qui ressort des travaux de STAHL et al. (I932), Fingerling (I933), Hutchinson et al., (I943), Grashuis et De Man (I947) ; Bifilinski et Bifinsska (I954), Laube et Weissbach (I96I-62). Woodman et Evans (I943), McMilifen et al. (r948), Husby et PRESTHEGGE (I952) ont montré également que des pommes de terre convenablement déshydratées peuvent se substituer aux céréales (orge), à quantité égale et suivant une proportion représentant $30 \mathrm{p}$. Ioo de la ration totale, sans qu'il en résulte des effets défavorables sur la vitesse de croissance, l'indice de consommation ou la qualité des carcasses. Notons cependant, d'après McMrLLEN et al., que cette utilisation est meilleure lorsque les animaux sont plus âgés (au-delà de $70 \mathrm{~kg}$ de poids vif) et lorsque le taux d'incorporation dans le régime est inférieur à 50 p. Ioo; au-delà il s'ensuit une diminution de la valeur de remplacement vis-à-vis de l'orge. ZAuscH et Holzschuch ( $\mathrm{I} 960$ ) rapportent de leur côté une mauvaise croissance et des troubles digestifs (diarrhée) avec un régime renfermant $40 \mathrm{p}$. Ioo de pommes de terre déshydratées. Plus récemment, HofmanN (I960, I965) enregistre avec des pommes de terre déshydratées des performances légèrement plus faibles qu'avec un aliment concentré complet ou des pommes de terre cuites; cependant, les écarts observés ne sont pas significatifs et doivent être attribués principalement au procédé de dessiccation employé.

Si l'on en juge d'après les résultats obtenus dans la présente expérience, on peut en déduire que les pommes de terre séchées à l'état de cossettes crues puis réduites en farine sont moins bien valorisées que les tubercules frais et cuits. Sans doute la consommation de matière sèche est-elle plus élevée qu'avec les pommes de terre fraîches, par suite de l'élimination de l'important volume d'eau contenu dans ces dernières, mais elle demeure plus faible que dans le lot témoin à base d'orge. Pour expliquer cette action dépressive des pommes de terre séchées sur l'appétit, phénomène observé également par d'autres auteurs, dont HoFMAnn (I960), on pourrait incriminer l'équilibre azoté de la ration. En effet, au cours de la déshydratation, il a été observé une chauffe irrégulière entraînant une calcination partielle du produit, et probablement une dénaturation plus ou moins importante des matières azotées. Ceci se traduit en particulier par une légère dépression de la digestibilité de ces dernières, ainsi que l'ont montré Woodmax et Evans (I939), Hutchinson et al. (I943), WOMlbier et NeCKERMANx (I952), Hommans (I965). Il est possible également que l'excès de chauffage incriminé ait fait apparaître dans le produit déshydraté des odeurs ou des saveurs peu plaisantes pour le porc. Sur le plan pratique, et compte tenu de la qualité du produit employé, il conviendrait ainsi d'élever légèrement le niveau de complémentation azotée. Par ailleurs, nous devons attribuer à ce traitement la diminution de la valeur énergétique du produit séché : $0,9 \mathrm{UF}$ par $\mathrm{kg}$ de matière sèche au lieu de I,I UF pour les tubercules frais. Les valeurs énergétiques correspondantes, exprimées en énergie digestible par $\mathrm{kg}$ de matière sèche, sont. respectivement $335^{2}$ et $376 \mathrm{r} \mathrm{kcal}$, contre $353 \mathrm{I} \mathrm{kcal}$ pour l'orge ; il est intéressant. de noter que cette valeur trouvée pour l'orge est assez proche de celle déterminée par NEHRING et ses collaborateurs (I963) soit 35 Io kcal digestibles par $\mathrm{kg}$ de matière 
sèche. Pour expliquer la perte d'énergie ainsi provoquée par la déshydratation, on pourrait invoquer une cuisson irrégulière de l'amidon. De nombreux auteurs, parmi lesquels BolimanN (I932), Frens (I943), BraUde et Mircherl (I95I), BrErrem et Husby (I95I), Leroy, Zelter et FÉvrier (I952), Martin et Buysse (I955), ont montré en effet que la valeur nutritive de la pomme de terre dépend étroitement de son degré de cuisson; au contraire, selon HoFmaNN (I960) l'amidon de pommes de terre crues puis séchées serait aussi bien utilisé que l'amidon cuit. Notons également que le produit utilisé dans la présente expérience a été obtenu à partir de tubercules non lavés et renfermant une teneur anormalement élevée en cendres ( 9 p. roo de la matière sèche contre $6 \mathrm{p}$. Ioo pour les tubercules frais et lavés), ce qui pourrait entraîner à la fois une diminution de la valeur énergétique proprement dite et une dépression de la digestibilité, laquelle est cependant moins marquée si on la rapporte à la matière organique. On peut donc présumer qu'une préparation correcte (lavage soigné suivi d'une déshydratation à une température modérée) permettrait de mieux valoriser le produit déshydraté et sans doute de lui donner une valeur comparable au produit cuit. C'est ce qui apparaît des travaux récents de KLUSMANN (I962), Teichmann (I962), Richter, Cranz et AN'Toni (ig62), Richter et Farries (Ig62), qui n'ont observé aucune différence dans l'utilisation des pommes de terre séchées ou cuites, selon que l'on considère la vitesse de croissance, l'indice de consommation de matière sèche, la digestibilité des éléments énergétiques et azotés, ou les caractéristiques de composition corporelle. Quoi qu'il en soit, si 1'on se réfère aux résultats obtenus par ailleurs avec les betteraves (RÉRAT et HENRY, I964), on peut penser que la valeur de remplacement des pommes de terre déshydratées serait sensiblement améliorée grâce à l'introduction dans le régime d'un taux plus faible que celui utilisé dans la présente étude.

$E n$ conclusion, les pommes de terre fraîchement cuites peuvent être introduites avantageusement dans le régime du porc en croissance, selon le système L FHMANN, grâce à un apport complémentaire de I $500 \mathrm{~g}$ d'un aliment concentré renfermant I7 p. Ioo de matières azotées totales (soit $300 \mathrm{~g}$ d'un concentré azoté dosant 44 p. Ioo de matières azotées et I $200 \mathrm{~g}$ d'orge). L'important volume d'eau contenu dans les tubercules constitue le facteur limitant de l'ingestion énergétique et par suite de la vitesse de croissance, comparativement à un régime exclusivement concentré à base d'orge. Quoi qu'il en soit, 1'utilisation globale de la ration est comparable et les carcasses obtenues sont plus maigres. La consommation de pommes de terre selon ce mode de rationnement représente environ $40 \mathrm{p}$. Ioo de la consommation totale de matière sèche.

L'emploi de pommes de terre déshydratées, à un même niveau de complémentation énergétique ( I $500 \mathrm{~g}$ d'aliment concentré), se traduit par des performances moindres que celles obtenues à partir de tubercules frais, tant en ce qui concerne la vitesse de croissance que l'indice de consommation ou la qualité des carcasses. La valeur de remplacement du produit obtenu est de $0,8 \mathrm{~kg}$ d'orge (ou UF) par $\mathrm{kg}$ contre $0,27 \mathrm{~kg}$ d'orge par $\mathrm{kg}$ de tubercules frais (soit respectivement 0,9 et I,I UF par $\mathrm{kg}$ de matière sèche). Cette diminution de la valeur nutritive du produit séché doit être attribuée en premier lieu à une mauvaise préparation (lavage insuffisant, température de dessiccation trop élevée). Remarquons par ailleurs que l'ingestion de pommes de terre déshydratées a été relativement importante, représentant $40 \mathrm{p}$. Ioo de la consommation totale de matière sèche $(28 \mathrm{p}$. Ioo entre 30 et $60 \mathrm{~kg}$ 
de poids vif et $48 \mathrm{p}$. Ioo entre 60 et Ioo $\mathrm{kg}$ ). C'est pourquoi il y a lieu de penser que les performances seraient sensiblement améliorées si l'on introduisait une proportion plus faible de pommes de terre déshydratées en remplacement de l'orge dans l'aliment concentré, par exemple respectivement ${ }_{5}$ et $30 \mathrm{p}$. Ioo au cours des périodes $30-60 \mathrm{~kg}$ et $60-\mathrm{I} 00 \mathrm{~kg}$ de poids vif. On pourrait également envisager une élévation du niveau de complémentation azotée, soit $350 \mathrm{~g}$ d'un concentré azoté renfermant $44 \mathrm{p}$. Ioo de matières azotées totales en complément de I $200 \mathrm{~g}$ d'orge ou I $500 \mathrm{~g}$ d'un aliment concentré renfermant I 8 à I 9 p. Ioo de matières azotées totales.

$$
\text { Rę̧u pour publication en mai } 1966 .
$$

\section{REMERCIEMENTS}

A l'Institut technique de la Pomme de Terre pour nous avoir fourni gracieusement les lots de pommes de terre et pour sa contribution à la réalisation de ces essais.

\section{SUMMARY}

\section{USE OF DRIED AND COOKED POTATOES IN COMPARISON TO BARLEY} IN FEEDING GROWING PIGS

The use of dried or cooked potatoes in the feeding of growing pigs, in comparison to barley, was the subject of two experiments, one with groups of pigs and one in digestibility crates. In the first experiment (A) three groups of $\mathrm{I}_{3}$ Large White pigs of initial liveweight $30 \mathrm{~kg}$ were fed to foo $\mathrm{kg}$ liveweight on the Lehmann system, in which they were given daily $300 \mathrm{~g}$ concentrate with $44 \mathrm{P}$. 100 crude protein and, for the respective groups :

- barley to appetite (Group I) ;

- I $200 \mathrm{~g}$ barley and dried potatoes to appetite (Group 2);

- I $200 \mathrm{~g}$ barley and freshly cooked potatoes to appetite (Group 3 ).

In the second experiment (B) 6 pigs were put in digestibility cages between 40 and $70 \mathrm{~kg}$ liveweight and were fed as the groups in the first experiment, in a Latin square design, in order to estimate the digestibility of energy and nitrogen of the feed by difference.

From the results obtained in the interval between 30 and $100 \mathrm{~kg}$ liveweight average daily gain in g per day and "index of consumption ", intake of dry matter per kg gained, were for groups I, 2 and 3 , respectively, $672 \mathrm{~g}$ and $3,35 \mathrm{~kg}, 568 \mathrm{~g}$ and $3,67 \mathrm{~kg}$ and $59^{2} \mathrm{~g}$ and $3,35 \mathrm{~kg}$. Corresponding values for criteria of carcase composition, carcase yield, percentage lean cuts (ham and loin) and fat cuts (back and belly) in the carcase and mean depth of back fat ( $\left.\frac{\text { kidney }+ \text { back }}{2}\right)$ in $\mathrm{mm}$ were, respectively: Group I, 72.3, 48.7, I9.8 and 30.7; group 2, 70.9, 50.0, 19.3 and 28.9; group $3,70.3,52.4$, I 7.1 and 26.2 . Percentage digestibility of energy and of crude protein and energy value expressed as kcal digestible energy per $\mathrm{kg}$ dry matter were for barley $77.2,65.3$ and $353 \mathrm{I}$, for dried potatoes $80.9,35.4$ and $335^{2}$ and for cooked potatoes $85.4,49.8$ and $376 \mathrm{I}$.

Giving fresh potatoes in the diet reduced growth rate compared with barley but the intake of dry matter per kg gained was comparable, and the carcases were leaner. With this method of feeding potatoes supplied $37 \mathrm{p}$. Ioo of the total intake of dry matter. In contrast, dried potatoes compared with fresh tubers reduced performance as regards growth rate, intake of dry matter per unit gained and carcase quality; between 30 and $100 \mathrm{~kg}$ liveweight the dried product supplied $40 \mathrm{p}$. 100 of the total intake of dry matter. The replacement value of dried potatoes was $0.8 \mathrm{~kg}$ barley (or feed unit) per $\mathrm{kg}$ against $0.27 \mathrm{~kg}$ barley per $\mathrm{kg}$ for fresh tubers, equivalent to 0.9 and r.I feed units per $\mathrm{kg}$ dry matter. However, the reduced feeding value of the dried potatoes compared with the fresh product could be considered at least in part due to faulty preparation; the potatoes were not washed, and they were overheated during drying. 


\section{RÉFÉRENCES BIBLIOGRAPHIQUES}

Armsby H. P., 1922. The nutrition of fam animals, $743 \mathrm{pp}$. The McMillan $\mathrm{Co}, \mathrm{N}$. Y.

Bielinski K., Bielinska K., I95\%. L'emploi de la pulpe de pommes de terre déshydratée dans l'alimentation du porc (en polonais). Racz. Nauk. rol. (B), 68, 297-308.

Bollmann W., 1932. Untersuchungen über die Verdaulichkeit der Nährstoffe von rohen, gedämpften und gedämpfteingesäuerten Kartoffeln am Schwein, am Hammel, an der Milchkuh und am Mastochsen. Ztschr. Züchtung, 24, 330-357.

Braude R., Mitchell K. G., i95 I. Potatoes for fattening pigs. Comparison of cooked and raw potatoes. Agriculture, 57, 501-506.

Breirem K., Husby M., i95I. Essais sur l'emploi de l'ensilage de pommes de terre cuites ou crues dans l'alimentation du porc (en norvégien). Norsk Landbruk, 17, 59-64;89-93.

EckноғF, 1963. Das Schwein und die Kartoffel. Schweinezucht und Schweinemast, 11 (3), 58-60.

Fender M., Kirsch W., FEwSON D., I963. Beitrag zur Hackfruchtmast mit Schwein unter besonderer Berücksichtigung das Nährstoff gehaltes der Beifuttermischungen und der Fütterungstechnik. Z. Tierphysiol. Tierernähr. Futtermittel,, 19, 175-180.

Fingerlang G., 1933. Der Nährwert von Kartoffelflocken und Kartoffelschnitzelı. Die Landw. Vers. Sta., 114, I-II 2 .

Frens A. MI, I943. Les propriétés dićtétiques défavorables des pommes de terre crues pour le porc en croissance (en néerlandais). Versl. Vereeniging Exploitatie Proefzinvelboerderij, Horn, $261-290$.

Friend D. W., Cunningham H. M., Nicholson J. W. G., ig63. The feeding value of dried potato pulp for pigs. Canad. J. Anim. Sci., 43, 24I-25I.

Grashuts J., De MaN T. T., I947. lessais complémentaires sur la valeur nutritive et l'emploi des cossettes de pommes de terre sécbées dans l'alimentation du porc (en néerlandais). Inst. Moderne Veevoeding "T'eschothorst ", Hoogland, $14 . \mathrm{pp}$

Hofmann P., I960. Schweinemastversuch mit Kartoffeltrockenschnitzeln und Kartoffelflocken. Der kartoffelban, 11, 6.

Hofman. P., 1965. Schweinemastversuch mit Kartoffeltrockenschnitzelin. Bayer Landw. Jb, 42, $339-347$.

Hoser S., 1965. Utilisation des pommes de terre ensilées pour l'engraissement des porcs (en polonais). Praglad Hodowlany, 34 (9), $17^{-1} 9$.

Hughes B. P., I958. The aminoacio composition of potato protein and of cooked potato. Brit. J. Nulr., 12, $188-195$.

Husby M., PRestinegge K., 1952. Le sorgho, les caroubes et la poudre de pomme de terre dans l'alimentation du porc (en norvégien). Landhrukshögsk Foringsforsök. Rapport no 26, p. 8.

Hutcininson J. C. D., Bacon J. S. D., Macrae T. F., Worden A. N., i9.3. The nutritive value of potato protein for the pig. Biochem. J., 37, 550-562.

Kastell A., 1957. Die quantitative Bestimmung der Aminosäuren in Futtermitteln. Das Kartoffel-eiweiss. Mikrochim. Acta, 689-698.

Kellner O., Just M., Eisenkolbe P., Poppe M., igo8. Untersuchungen über die Verdaulichkeit getrochkneter Kartoffeln. Landw. Vers. Sla., 68, 39-60.

Kellner O., Neumann R., igio. Fütterungsversuche mit Schweinen über die Verdaulichkeit getrockneter Kartoffeln und des entfetteten Sojabohnenmehls. Land. Vers. Sta., 73, 235-240.

Klusmann W., 1962. Der kartoffelBau., 13, I 59-160.

Lantzsch H. J., GutTe J. O., Molnar S., Lenkeit W., I964. Beitrag zur Fragel der Fütterungsintensität und Beifuttermenge in der Hackfruchtmast derSchweine. Z. Tierphysiol. Tierernähr. Futlermiltelk.,19, $357^{-363}$.

LAUBE W., WeISSBACH F., I961-62. Untersuchungen an Schweinen über Schmackhaftigkeit, Bekömmlichkeit, Verdaulichkeit und Mastwirkung von walzengetrockneten Kartoffeleiweiss. Jahr. Arbeitsgemeinschaft Fütlerungsberatung, 4, $24 \mathrm{I}-252$.

Lehmann F., 1929. Tierische mehle und Futtermittel aus niederen Tieren. In Mangolg E. Nährstoffe und Futtermitlel (1), 498-527, Springer, Berlin.

Leroy A. M., r965. Influence de la matière azotée sur la dépense énergétique des animaux. World. Rev. Anim. Prod. (3), 3I-4I.

Leroy A. M., Zelter S., Février R., i952. L'utilisation de la pomme de fterre pour l'alimentation des animaux domestiques. Ann. Zootech., 1, 87-145.

Mangold E., Columbus A., I938. Verdaulichkeit und biologische Wertigkeit von Kartoffeleiveiss beim Schwein. Landw. Vers Sta., 129, 12-27.

Martin J., Buysse F., 1955. La pomme de terre dans l'alimentation du porc. Comparaison entre les pommes de terre étuvées et les pommes de terre crues réduites en purée. Rez. Agric., Bruxelles, 8, II35-II58.

Mc Millen W. N., Brown G. A., Luecke R. W., I948. Dehydrated potatoes for swine. Quart. Bull. Michigan Agric. Expt. Sta., 30, 380-382. 
Neiring K., Hoffmann L., Schiemann R. I963. Die energetische Verwertung der Futterstoff 2.3 . Die energetische Verwertung der Kraftfutterstoffe durch Schweine. Arch. Tierernähr., 13, I47-I6I.

Nehring F., Schiemann R., Hoffmann L., Klippel W., i960. Die Verwertung der Futterenergic in Abhängigkeit vom Emähungsniveau. 2. Versuche mit Schweinen. Arch. Tierernähr, 10, 275-320.

Pieper E., 1962. Kartoffelbedarf je Mastschwein. Teerzüchter, 14, 8ro-812.

Rérat A., Henry Y., Ig64. Utilisation de la betterave "danoise "dans l'alimentation du porc en croissance. I. Recherche d'un plan de rationnement. Ann. Zootech., 13, 21 7-236.

RÉrat A., Henry Y., I965a. Utilisation de la betterave "danoise " dans l'alimentation du porc en croissance. II. Variations du plan de rationnement et du poids d'abattage. Ann. Zootech., 14, $371-377$.

Rér AT A., Henry Y., I $965 b$. Utilisation de la betterave "danoise "dans l'alimertation du porc en croissance. III. Valeur fourragère et digestibilité. Ann. Zootech., 14, 379-389.

Richter K., Cranz K. L., Oslage H. J., I956. Vergleichende Untersuchungen über den Einfluss einer unterschiedlichen Fütterung von Schweinen mit Getreide, Kartoffeln, und Zuckerrüber auf Mastenturcklung und Schlachtqualität. Zuchtungskunde, 28, 93-104.

Richter K., Cranz K. L., Antoni J. J., 1962. Futterwert und Futterwirkung von im Trommeltrockner hergerstellten Schnitzeln. Der Karioffel Bau, 13 (9).

Richter K., FARries F. E., I962. Zusammensetzung, Verdaulichkeit und Nährwert von Kartoffelschnitzeln. Der kartoffel Bau, 13 (I I).

Schuphan W., 1952. Studien über essentielle Aminosäueren in Kartoffeln. 2. Die biologische Eiweisswertigkeit der Kartoffel (Solanum tuberosum I.) im Ernährungsversuch und Spiegel der essentieller Aminosäuren. Qual. Plant. Materize vegetabiles, 6, I6-38.

SEEFELDT G., I962. Richtigen Eiisatz der Kartoffel im Schweinestall. Schweinezucht. Schweinemast., 10, 2 I9-220.

SNEDECOR G. W., 1956. Statistical methods applied to experiments in agriculture and biology, $5^{\mathrm{e}}$ éd., $534 \mathrm{p}$. The Iowa state Univ. Press, Ames, Iowa.

Stahl W., Göhner A., BARTh F., I932. Welchen wert hat Kartoffelpülpe für die Schweinemast? Zeitschr. Schweinezucht, 39, 229-23I.

Stahl W., Freidrich E., I 940. Trocken kartoffelshrot (Verfahren Ing. Maus) im Vergleich zu Kartoffel Hocken. J. ber. Landw., 87, $243^{-248 .}$

Teichmann W., I962. Der Kartoffel Bau, 13, I60-161.

Weniger J. H., I957. Weitere Untersuchungen über das Verhalten des Magen Darmkanals des Schweines unter dem Einfluss Verschiedenartiger Frnăhrung. Arch. Tierernähr, 6, 223-230.

Wohlbier W., Neckermani L., 1952. Der Nährwert von Kartoffelflocken und Press Kartoffeln, nach versuchen an Schweinen. Arch. Tieremähr, 2, ı66-г 76.

Woodman H. L., Evans R. E., 1939. The composition and digestibility, when fed to pigs and sheep, of potato cossettes and potato meal. J. agric. Sci., 29, 347-363.

Woodman H. E., Evans R. E., 1943. Further investigations of the feeding value of artificially dried potatoes, the composition and nutritive value of potato cossettes, potato meal, potato flakes, potato slices and potato dust. J. agric. Sci., 33, I-14.

ZaUsch M., Holzschuh W., I960-196x. Getrocknete Kartoffeln bei der Schweinefütterung. Jahrb. Arbeitsgemeinschaft. Fïlterungsberatung., 3, I $73^{-1} 82$. 